\title{
A STUDY OF THE WAVES AND BOUNDARY LAYERS DUE TO A SURFACE PRESSURE ON A UNIFORM STREAM OF A SLIGHTLY VISCOUS LIQUID OF FINITE DEPTH
}

\author{
ARGHYA BANDYOPADHYAY
}

Received 31 May 2006; Revised 25 October 2006; Accepted 22 November 2006

The $2 \mathrm{D}$ problem of linear waves generated by an arbitrary pressure distribution $p_{0}(x, t)$ on a uniform viscous stream of finite depth $h$ is examined. The surface displacement $\zeta$ is expressed correct to $O(\nu)$ terms, for small viscosity $\nu$, with a restriction on $p_{0}(x, t)$. For $p_{0}(x, t)=p_{0}(x) e^{i \omega t}$, exact forms of the steady-state propagating waves are next obtained for all $x$ and not merely for $x \gg 0$ which form a wave-quartet or a wave-duo amid local disturbances. The long-distance asymptotic forms are then shown to be uniformly valid for large $h$. For numerical and other purposes, a result essentially due to Cayley is used successfully to express these asymptotic forms in a series of powers of powers of $v^{1 / 2}$ or $v^{1 / 4}$ with coefficients expressed directly in terms of nonviscous wave frequencies and amplitudes. An approximate thickness of surface boundary layer is obtained and a numerical study is undertaken to bring out the salient features of the exact and asymptotic wave motion in question.

Copyright (c) 2006 Arghya Bandyopadhyay. This is an open access article distributed under the Creative Commons Attribution License, which permits unrestricted use, distribution, and reproduction in any medium, provided the original work is properly cited.

\section{Introduction}

The classical problem of waves due to any initial variable pressure distribution on the surface of an ocean of finite depth has attracted the attention of many researchers mainly because of its connection with several important physical problems such as ship-waves, impact of aerial blast waves on the ocean, storms, tsunamis, and so forth. Although the physical settings of these problems are different, there exists a similarity in the analyses of the wave integrals and also of the wave behavior in many cases. For example, the linear waves generated by initial surface disturbances in an inviscid liquid exhibits the same qualitative behavior as that of the tsunamis (cf. Wehausen and Laitone [11, page 618]). Our objective here, however, is to study the effect of viscosity on the waves in question without any further elaboration of such interrelationships. 
It is well known that while the formal solution of the linear problem of waves produced by surface disturbances on a stationary or moving viscous fluid of finite depth is easily obtained, substantial difficulty arises in evaluating or analyzing this solution which can be seen in the work of Bandyopadhyay [1], Nikitin and Potetyunko [6], and Pramanik [7]. Such analyses have so far remained confined to derivation of an asymptotic expression of the surface displacement $\zeta$ for small viscosity $\nu$ at large times and distances, and there seems to have no attempt at analytical and numerical studies of the wave integrals in its exact form (I). Also the approximation of $\zeta$ for smallness of $\nu$ is generally carried out in a rather heuristic way without paying attention to the restriction that may have to be placed on the pressure models for the approximation to succeed (II).

In this paper, we examine the two-dimensional form of the problem stated above for a uniformly flowing stream of small viscosity, our main objective being to find the restriction mentioned in (II) for a general pressure model $p_{0}(x, t)$ and to achieve (I) for a special model $p_{0}(x) e^{i \omega t}$. For the former, we first express $\zeta$ correct to $O(\nu)$ terms by a proven approximation formula of Bandyopadhyay [1], and show incidentally that an $O(\sqrt{v})$ analysis of $\zeta$ (cf. Pramanik [7] for $p_{0}(x) e^{i \omega t}$ ) fails to bring out the effect of viscosity on $\zeta$ for large depths, while an $O(\nu)$ analysis succeeds in correcting this shortcoming (cf. (3.9) together with (3.7), (3.8), and (3.10)). Secondly, in Section 4 we initiate the study (I) for the special pressure distribution $p_{0}(x) e^{i \omega t}$, and verify that a steady-state exists everywhere and not merely for large distances (contrast Pramanik [7]). This steady state as it is shown in Section 4.2 consists of a propagating wave system $\zeta_{P}^{*}$ compounded by standing local disturbances for all times and distances. After the exact evaluation of the former, we find that this system of propagating waves $\zeta_{P}^{*}$ is either a damped and constant-velocity waveduo or a wave-quartet according to the fact that the stream speed $u_{0}$ does or does not exceed a certain critical value. At large distances, the standing waves fade out in comparison to the leading term, denoted by $\zeta^{* *}$, of the asymptotic expression of $\zeta_{P}^{*}$. When $\zeta^{* *}$ is retained correct up to $O(\sqrt{v})$ terms, it agrees with the corresponding result obtained by Pramanik [7]; this expression, however, as is shown here makes the long-distance wave form inaccurate for large $h$, while in our case the presence of $O(\nu)$ terms in $\zeta_{P}^{*}$ rectifies such inappropriate result for large depth (Section 5).

We next show (Sections 6.1 and 6.2) that it is advantageous to write $\zeta^{* *}$ in the following form by a result essentially due to Cayley (Whittaker and Watson [12, page 147]):

$$
\zeta^{* *}= \begin{cases}\zeta_{0}+\sqrt{\nu} \zeta_{2}+\nu \zeta_{4}, & \text { when } u_{0} \neq u_{0}^{*}, \\ \zeta_{0}^{\prime}+\nu^{1 / 4} \zeta_{1}^{\prime}+\nu^{1 / 2} \zeta_{2}^{\prime}+\nu^{3 / 4} \zeta_{3}^{\prime}+\nu \zeta_{4}^{\prime}, & \text { when } u_{0}=u_{0}^{*},\end{cases}
$$

where each $\zeta_{j}$ (or $\zeta_{j}^{\prime}$ ) is wave-quartet or wave-duo, and in either case the wave amplitudes and wave frequencies are all directly expressed in terms of their nonviscous values. Such expansions also facilitate a numerical study of the wave motion. Some interesting features of the wave motion are brought out through a computational study of the exact wave integral $\zeta_{P}^{*}$ and its asymptotic forms which are discussed in Section 8. Finally, since the motion may be considered to have a high Reynolds number $\sqrt{g h^{3}} / \nu$, a question naturally arises as to the formation of boundary layers both at the surface and the bottom and the shapes they assume vis-à-vis those of waves. In this regard, in Section 7, we determine the 
cross-vertical vorticity $\omega_{z}(x, y, t)$ and find its approximate value correct to $O(\nu)$ terms. Its Cayley expansion is next derived for $|x| \rightarrow \infty$ by the same procedure as adopted for $\zeta^{* *}$. These expressions are utilized for the determination of the approximate thickness of the boundary layer at the surface at large horizontal distances from the source.

In passing, mention may be made of two important papers, one by Beale [2] who is concerned about the existence of solution of the above problem in a general threedimensional nonlinear setting when the initial surface and velocity fields are prescribed, and the other paper by Ehrenmark and Body [4] who are however interested in the solution of the inviscid problem in the presence of a plane beach under the varying conditions on the surface as well as on the bed.

\section{The problem and its integral solution}

For the two-dimensional motion of a horizontally unbounded viscous liquid of constant depth $h$ parallel to the $x y$-plane, a coordinate system is chosen such that the origin is in the undisturbed free surface which is the $x y$-plane; the $y$-axis is vertically upwards and the $x$-axis is positive to the right, while the stream moves in the $O x$ direction with speed $u_{0}$ relative to the frame of reference.

Waves of height $\zeta(x, y)$ above the undisturbed surface are generated by the action of the pressure distribution $p_{0}(x, t)$ which is suddenly applied on $y=0$ at $t=0 . p_{0}(x, t)$ is assumed to have a Fourier transform in $x$ and also to have a Laplace transformation in $t$. If $\zeta$ and the disturbance velocity components $(u, v)$ parallel to the axes are small, the equation of continuity and the linearized Stokes-Navier equations are, respectively,

$$
\begin{gathered}
\frac{\partial u}{\partial x}+\frac{\partial v}{\partial y}=0 \\
\left(\frac{\partial}{\partial t}+u_{0} \frac{\partial}{\partial x}\right)(u, v)=-\frac{1}{\rho}\left(\frac{\partial}{\partial x}, \frac{\partial}{\partial y}\right) p+\nu \nabla^{2}(u, v),
\end{gathered}
$$

where $p=p_{1}+\rho g y, \nabla^{2} \equiv \partial^{2} / \partial x^{2}+\partial / \partial y^{2},-\infty<x<\infty,-h \leq y \leq \zeta \approx 0, t \geq 0$. For a fluid at rest, the initial conditions are

$$
u=v=0, \quad \zeta=0 \quad \text { for } t=0
$$

The linearized normal stress and the no-shear stress condition on the surface together with the surface boundary condition are, respectively,

$$
-p_{0}(x, t)=-p+\rho g \zeta+2 \mu v_{y}, \quad u_{y}+v_{x}=0, \quad \zeta_{t}+u_{0} \zeta_{x}=v \quad \text { for } y=0, t>0 .
$$

The condition of no-slip at the bottom is

$$
u=v=0 \quad \text { for } y=-h, t>0
$$

Lastly the conditions at infinity are $u, v, p$ and their $x$-derivatives are zero as $|x| \rightarrow \infty$. 
4 A study of the waves and boundary layers

Here subscripts $t, x, y$ refer to partial differentiation. Our main interest is in the expression for $\zeta$.

For convenience, we will suppress arguments of the functions and their transforms in general. Let $U, V, H, P, P_{0}$ denote the Fourier transforms of $u, v, \zeta, p, p_{0}$, respectively, in $x$, and let $\tilde{U}, \tilde{V}, \tilde{H}, \widetilde{P}, \widetilde{P}_{0}$ denote the Laplace transforms of the former in $t, k$, and $s$ being the respective transform parameters.

The formal solution of the problem may be written as

$$
[u, v, p, \zeta]=\frac{1}{(2 \pi)^{3 / 2} i} \int_{-\infty}^{\infty} e^{-i k x} d k \int_{c-i \infty}^{c+i \infty}[\tilde{U}, \tilde{V}, \tilde{P}, \tilde{H}] e^{t s} d s
$$

where the constant $c$ has its usual meaning, and the transform integrals are given by

$$
\begin{gathered}
\tilde{U}=\frac{i \operatorname{sgn} k}{\rho\left(s-i k u_{0}\right)}[|k| \tilde{A}\{\cosh |k|(y+h)-\cosh m(y+h)\} \\
+\widetilde{B}\{|k| \sinh |k|(y+h)-m \sinh m(y+h)\}], \\
\tilde{V}=\frac{|k|}{\rho\left(s-i k u_{0}\right) m}[\widetilde{A}\{|k| \sinh m(y+h)-m \sinh |k|(y+h)\} \\
+m \tilde{B}\{\cosh m(y+h)-\cosh |k|(y+h)\}], \\
\tilde{H}=-|k|\left\{\mu D\left(s-i k u_{0}\right) m\right\}^{-1}(m \sinh |k| h \cosh m h-|k| \sinh m h \cosh |k| h), \\
D \widetilde{A}=\left(k^{2}+m^{2}\right) \cosh m h-2 k^{2} \cosh |k| h, \\
m D \tilde{B}=2 m k^{2} \sinh |k| h-|k|\left(k^{2}+m^{2}\right) \sinh m h, \\
-\widetilde{P}_{0}\left(s-i k u_{0}\right) D v^{-1}=4 k^{2}\left(k^{2}+m^{2}\right)-\cosh m h \cosh |k| h\left\{\left(k^{2}+m^{2}\right)^{2}+4 k^{4}+\frac{\sigma^{2}}{v^{2}}\right\} \\
+|k| m^{-1} \sinh m h \sinh |k| h\left\{\left(k^{2}+m^{2}\right)^{2}+4 k^{2} m^{2}+\left(\frac{g k}{\sigma v}\right)^{2}\right\},
\end{gathered}
$$

where

$$
\sigma^{2}=[\sigma(k)]^{2}=g|k| \tanh |k| h, \quad m^{2}=k^{2}+\frac{s}{v}-\frac{i k u_{0}}{v} .
$$

By (2.6), we have

$$
\tilde{H}=\frac{|k| \tilde{P}_{0}}{\rho} \frac{F\left(\nu^{1 / 2}\right)}{f\left(\nu^{1 / 2}\right)}, \quad-\widetilde{P}_{0}=-P+\rho g \tilde{H}+2 \mu \tilde{V}_{y},
$$


where

$$
\begin{aligned}
F\left(\nu^{1 / 2}\right) & =\left(s-i k u_{0}+v k^{2}\right)^{1 / 2} \tanh |k| h-v^{1 / 2}|k| \tanh \left(\frac{s-i k u_{0}+v k^{2}}{v}\right)^{1 / 2} h, \\
f\left(\nu^{1 / 2}\right)= & 4\left(s-i k u_{0}+v k^{2}\right)^{1 / 2}\left(s-i k u_{0}+2 v k^{2}\right) \nu k^{2} \operatorname{sech}|k| h \operatorname{sech}\left(\frac{s-i k u_{0}+v k^{2}}{v}\right)^{1 / 2} h \\
& -\left(s-i k u_{0}+v k^{2}\right)^{1 / 2}\left\{\left(s-i k u_{0}+2 v k^{2}\right)^{2}+4 v^{2} k^{4}+\sigma^{2}\right\}+|k| \nu^{1 / 2} \\
& \times\left\{\left(s-i k u_{0}+2 v k^{2}\right)^{2}+4 v k^{2}\left(s-i k u_{0}+v k^{2}\right)+g|k| \operatorname{coth}|k| h\right\} \\
& \times \tanh |k| h \times \tanh \left(\frac{s-i k u_{0}+v k^{2}}{v}\right)^{1 / 2} h .
\end{aligned}
$$

By the inversion theorem for Laplace transform and the convolution theorem, one then gets

$$
H=\frac{|k|}{2 \pi i \rho}\left[\int_{0}^{t} P_{0}(k, t-\tau) d \tau \int_{c-i \infty}^{c+i \infty} e^{s \tau} \frac{F\left(\nu^{1 / 2}\right)}{f\left(\nu^{1 / 2}\right)} d s\right] .
$$

The Fourier inversion theorem now gives the exact integral expression for $\zeta$,

$$
\zeta=\frac{1}{(2 \pi)^{3 / 2} i \rho} \int_{-\infty}^{\infty}\left[\int_{0}^{t} P_{0}(k, t-\tau) d \tau \int_{c-i \infty}^{c+i \infty} e^{s \tau} \frac{F\left(\nu^{1 / 2}\right)}{f\left(\nu^{1 / 2}\right)} d s\right]|k| e^{-i k x} d k .
$$

The corresponding integral expressions for $u, v, p$ follow in a similar way.

\section{Waves in a slightly viscous fluid}

If the kinematic coefficient of viscosity $v$ of the fluid is small, it may be shown that

$$
\begin{aligned}
\zeta= & -\frac{1}{(2 \pi)^{3 / 2} i \rho} \int_{-\infty}^{\infty}|k| e^{-i k x} d k\left[\int_{0}^{t} P_{0}(k, t-\tau) \times e^{i k u_{0} \tau} d \tau \int_{c-i \infty}^{c+i \infty} e^{s^{\prime} \tau}\left(\frac{T_{1}}{\Delta_{1}}\right) d s^{\prime}\right] \\
& +O\left(\nu^{3 / 2}\right), \quad \text { as } \nu \longrightarrow 0,
\end{aligned}
$$

provided as $k \rightarrow \infty, P_{0}\left(k_{1}, k_{2}, \tau\right)$ is of the order $O\left(k^{-17 / 4-n}\right), n>0$, uniformly in $\psi$, and $\tau$ with $k_{1}=k \cos \psi, k_{2}=k \sin \psi[11]$.

Here $s^{\prime}=s-i k u_{0}$, and

$$
\begin{gathered}
T_{1} \equiv T_{1}\left(s^{\prime}, k\right)=s^{\prime} \tanh |k| h-|k|\left(\nu s^{\prime}\right)^{1 / 2}+\frac{1}{2} \nu k^{2} \tanh |k| h, \\
\Delta_{1} \equiv \Delta_{1}\left(s^{\prime}, k\right)=s^{\prime 3}+s^{\prime} \sigma^{2}-\left(\nu s^{\prime}\right)^{1 / 2} g^{-1}\left(s^{\prime 2} \sigma^{2}+g^{2} k^{2}\right)+\frac{1}{2} \nu k^{2}\left(9 s^{\prime 2}+\sigma^{2}\right) .
\end{gathered}
$$


This approximation process leaves the conditions unaffected since we have from (2.5), (2.8), and Sneddon [9, page 184] that

$$
\begin{aligned}
p(x, 0,0+) & =\frac{1}{\sqrt{2 \pi}} \int_{-\infty}^{\infty} \lim _{s \rightarrow \infty}\left[s\{\tilde{P}(k, 0, s)\}_{(F / f) \rightarrow\left(-T_{1} / \Delta_{1}\right)}\right] e^{-i k x} d k \\
& =\frac{1}{\sqrt{2 \pi}} \int_{-\infty}^{\infty} \lim _{s \rightarrow \infty}\left[s\left\{\tilde{P}_{0}(k, s)\right\}_{(F / f) \rightarrow\left(-T_{1} / \Delta_{1}\right)}\right] e^{-i k x} d k \\
& =\frac{1}{\sqrt{2 \pi}} \int_{-\infty}^{\infty} P_{0}(k, 0) e^{-i k x} d k=p_{0}(x, 0) .
\end{aligned}
$$

Evaluation of the $s^{\prime}$-integral (3.1). The $s^{\prime}$-integrand in (3.1) has a branch point at $s^{\prime}=0$ as well as possible poles arising from those zeros of $\Delta_{1}$ for which $T_{1} \neq 0$. We suppose that the $s^{\prime}$-plane is cut along the negative real axis from 0 to $-\infty$, and that $s^{\prime 1 / 2}$ is real and positive when $s^{\prime}$ is real and positive. Considered as a sixth-degree polynomial in $s^{\prime 1 / 2}, \Delta_{1}$ has, for $\nu=0$, a double zero at the point zero and four other simple zeros at $\sqrt{\sigma} e^{ \pm i \pi / 4}$, $\sqrt{\sigma} e^{ \pm 3 i \pi / 4}$.

For the three corresponding values of $s^{\prime}$, namely

$$
s_{00}^{\prime}=0, \quad s_{10}^{\prime}=i \sigma, \quad s_{20}^{\prime}=-i \sigma,
$$

we may assume the following expansion for the possible zeros $s_{j}^{\prime}, j=0,1,2$ of $\Delta_{1}$ in powers of $v^{1 / 2}$ :

$$
s_{j}^{\prime}=s_{j 0}^{\prime}+s_{j 1}^{\prime} \nu^{1 / 2}+s_{j 2}^{\prime} \nu+s_{j 3}^{\prime} \nu^{3 / 2}+\cdots
$$

It is then found that

$$
s_{01}^{\prime}=0, \quad s_{02}^{\prime}-s_{02}^{\prime 1 / 2}\left(\frac{k}{\tanh k h}\right)+\frac{1}{2} k^{2}=0 .
$$

To our degree of accuracy, this shows that $T_{1}\left(s_{0}^{\prime}, k\right)=T_{1}\left(\nu s_{02}^{\prime}, k-\right)=0$.

Leaving aside then the zero $s_{0}$ of $\Delta_{1}$ and determining the other coefficients $s_{j e}$ for $\ell=1,2,3$, we find that the $s^{\prime}$-integrand of (3.1) has two simple poles at $s^{\prime}=s_{j}^{\prime}, j=1,2$, given by

$$
\begin{aligned}
s_{j}^{\prime}= & \alpha_{j}+\frac{1}{2}\left[1+(-1)^{j} i\right] \nu^{3 / 2}(2 \sigma)^{-1 / 2}|k|^{3} \operatorname{cosech}^{3} 2|k| h \\
& \times\left(\frac{-13}{4}+\frac{1}{2} \cosh 4 k h-\cosh 6 k h\right)+\cdots,
\end{aligned}
$$

where

$$
\begin{aligned}
\alpha_{j}= & \alpha_{j}(k)=(-1)^{j-1} i \sigma-\left\{1+(-1)^{j-1} i\right\}|k|\left(\frac{\sigma \nu}{2}\right)^{1 / 2} \operatorname{cosech} 2|k| h \\
& -2 v k^{2} \frac{\cosh 2 k h+\cosh 4 k h-1}{\cosh 4 k h-1}, \quad j=1,2 .
\end{aligned}
$$

(cf. Biesel's result cited by Wehausen and Laitone in [11, page 644].) 
Using a modified Bromwich contour (Tranter [10]) now completes the $s^{\prime}$-integration of (3.1) and the result is

$$
\zeta=-\frac{1}{\sqrt{2 \pi} \rho} \int_{-\infty}^{\infty}|k| e^{-i k x}\left[\int_{\tau=0}^{t} P_{0}(k, t-\tau) \times e^{i k u_{0} \tau} f_{2}(k, \tau) d \tau\right] d k,
$$

where

$$
f_{2}(k, t)=\sum_{j=1}^{2} \frac{T_{1}\left(s_{j}^{\prime}, k\right)}{\Delta_{1}^{\prime}\left(s_{j}^{\prime}, k\right)} e^{s_{j}^{\prime} \tau}-\frac{1}{2 \pi i} \int_{0}^{\infty} e^{-t \chi}\left\{\left[\frac{T_{1}}{\Delta_{1}}\right]_{s^{\prime}=\chi e^{i \pi}}-\left[\frac{T_{1}}{\Delta_{1}}\right]_{s^{\prime}=\chi e^{-i \pi}}\right\} d \chi .
$$

Introducing conditions similar to those of evaluating (3.1), we may show that the use of the values $s_{j}^{\prime}$ (given by (3.7)) instead of $s^{\prime}=\alpha_{j}$ for the roots would add only a term of order $\nu^{3 / 2}$ to $\zeta$ as calculated on the basis of (3.9) and (3.10), correct to the first power of $\nu$ in the amplitude. We also have

$$
\sum_{j=1}^{2} \frac{T_{1}\left(s_{j}, k\right)}{\Delta_{1}^{\prime}\left(s_{j}, k\right)} e^{s_{j} t}=(g|k|)^{-1} e^{-\sigma_{2} t}\left[\left(\sigma-\frac{\sigma_{1}}{2}\right) \sin \left(\sigma-\sigma_{1}\right) t+\frac{\sigma_{2}}{2} \cos \left(\sigma-\sigma_{1}\right) t\right],
$$

where

$$
\sigma_{1}=|k|\left(\frac{\sigma \nu}{2}\right)^{1 / 2} \operatorname{cosech} 2|k| h, \quad \sigma_{2}=\sigma_{1}+2 \nu k^{2}\left(1+\frac{\cosh 2 k h}{\cosh 4 k h-1}\right) .
$$

The final expression for $\zeta$ results when $f_{2}(k, t)$ in (3.10) is modified in accordance with (3.11) and (3.12). The surface displacement thus consists of damped gravity waves corresponding to the part of (3.9) containing the integral free term of (3.10) together with a

diffusive disturbance pattern corresponding to the remaining part of (3.9). We also note that in the former part, there also appears to be a phase lag as we combine the sine and cosine terms into a single sine or cosine term (cf. Prosperetti [8]).

\section{General character of the steady-state surface motion for an oscillatory pressure distribution}

When the applied pressure distribution is of the form $p_{0}(x, t)=p_{0}(x) e^{i \omega t} H(t), H(t)$ is the Heaviside unit function, we have by (3.11)-(3.12) that

$$
\begin{aligned}
\zeta= & -\frac{1}{\sqrt{2 \pi} \rho g} e^{i \omega t} \int_{-\infty}^{\infty} P_{0}(k) e^{-i k x} \frac{K(k)}{2} d k \int_{0}^{t} \exp \left[i\left(\sigma-\sigma_{1}\right)-i \omega+i k u_{0}-\sigma_{2}\right] \tau d \tau \\
& -\frac{1}{\sqrt{2 \pi} \rho g} e^{i \omega t} \int_{-\infty}^{\infty} P_{0}(k) e^{-i k x} \frac{\bar{K}(k)}{2} d k \int_{0}^{t} \exp \left[-i\left(\sigma-\sigma_{1}\right)-i \omega+i k u_{0}-\sigma_{2}\right] \tau d \tau \\
& +\frac{e^{i \omega t}}{\rho i(2 \pi)^{3 / 2}} \int_{-\infty}^{\infty} P_{0}(k)|k| e^{-i k x} d k \int_{0}^{t} e^{\left(-i \omega+i k u_{0}-\chi\right) \tau} d \tau \\
& \times \int_{0}^{\infty}\left\{\left[\frac{T_{1}}{\Delta_{1}}\right]_{s^{\prime}=\chi e^{i \pi}}-\left[\frac{T_{1}}{\Delta_{1}}\right]_{s^{\prime}=\chi e^{-i \pi}}\right\} d \chi .
\end{aligned}
$$


8 A study of the waves and boundary layers

Here

$$
K(k)=\frac{\sigma_{2}}{2}-i\left(\sigma-\frac{\sigma_{1}}{2}\right)
$$

Following the evaluation of the $\tau$-integrals in the first two terms of (4.1), we apply Watson's lemma (see Bleistein and Handelsman [3, page 103]) to the resulting $k$-integral to obtain its limit as $t \rightarrow \infty, x$ remaining fixed. For the third term of (4.1), we have

$$
\begin{aligned}
C_{3}= & \int_{-\infty}^{\infty} P_{0}(k)|k| d k \int_{0}^{1} t e^{\left(-i \omega+i k u_{0}\right) t \tau^{\prime}} d \tau^{\prime} \\
& \times \int_{0}^{\infty} \frac{\chi^{5 / 2} \nu^{1 / 2} \operatorname{sech}^{2} k h\left[\chi-(1 / 2) \nu k^{2}\left(8 \cosh ^{2} k h+1\right)\right] e^{-\chi t \tau^{\prime}} d \chi}{\Delta_{1}\left(\chi e^{i \pi}, k\right) \cdot \Delta_{1}\left(\chi e^{-i \pi}, k\right)} .
\end{aligned}
$$

Considering $\Delta_{1}\left(s^{\prime}, k\right)$ as a sixth-degree polynomial in $\sqrt{s^{\prime}}$, we may approximately express the product $\left.\left.\Delta_{1}\right]_{\chi e^{i \pi}} \cdot \Delta_{1}\right]_{\chi e^{-i \pi}}$ as

$$
\left(\chi+a_{o}^{2}\right)\left(\chi+a_{o}^{\prime 2}\right)\left(\chi+\alpha_{1}^{\prime}\right)^{2}\left(\chi+\alpha_{2}^{\prime}\right)^{2},
$$

where $a_{o}, a_{o}^{\prime}$ are the two values of $\sqrt{v} x$, where $x$ is given by $x^{2}-x(k / \tanh k h)+(1 / 2) k^{2}=0$ and $\alpha_{1}^{\prime}, \alpha_{2}^{\prime}$ are the same as $\alpha_{1}, \alpha_{2}$ of (3.8) but correct only up to the term in $\sqrt{\nu}$ therein. It is easy to see that $t \times \chi$-integral tends to zero as $t \rightarrow \infty$ for $0<\varepsilon<\tau^{\prime} \leq 1$, while the continuity of the $\chi-\tau^{\prime}$ integral in $0 \leq \varepsilon<1$ ensures that

$$
C_{3} \longrightarrow 0 \quad \text { as } t \rightarrow \infty \text {. }
$$

The steady-state surface displacement $\zeta^{*}$ thus comes out to be

$$
\zeta^{*} \sim \frac{e^{i \omega t}}{\sqrt{2 \pi} \rho g}\left(C_{1}+C_{2}\right)
$$

where

$$
\begin{aligned}
& C_{1}=\int_{-\infty}^{\infty} P_{0}(k) \frac{\left(\sigma-\sigma_{1} / 2\right)+i \sigma_{2} / 2}{2\left(-\sigma+\sigma_{1}+\omega-k u_{0}-i \sigma_{2}\right)} e^{-i k x} d k \\
& C_{2}=\int_{-\infty}^{\infty} P_{0}(k) \frac{-\left(\sigma-\sigma_{1} / 2\right)+i \sigma_{2} / 2}{2\left(\sigma-\sigma_{1}+\omega-k u_{0}-i \sigma_{2}\right)} e^{-i k x} d k .
\end{aligned}
$$

The picture of the ocean surface presented by $\zeta^{*}$ is, as will be shown below, one of a damped propagating plane wave system proceeding amid local standing disturbances at any distance which need not necessarily be far away from the origin. This view necessitates the identification of the infinities of the integrands, of $C_{1}$ and $C_{2}$, or more precisely, the zeros of the denominators $D_{\mp}$ of these integrands, which are pertinent to our assumption 
of small $v$,

$$
\begin{aligned}
& D_{-}=\omega-k u_{0}-2 i v k^{2}\left(1+\frac{\cosh 2 k h}{\cosh 4 k h-1}\right)-(i-1) \sigma_{1}-\sigma=0, \\
& D_{+}=\omega-k u_{0}-2 i v k^{2}\left(1+\frac{\cosh 2 k h}{\cosh 4 k h-1}\right)-(i+1) \sigma_{1}+\sigma=0 .
\end{aligned}
$$

Such zeros may be obtained in the form of perturbation expression of (4.9), (4.10) in positive powers of $\nu$ with the corresponding zeros of $\left(D_{\mp}\right)_{\nu=0}$ as initial values.

4.1. Zeros of $D_{\mp}$ for small $\nu$. First we note the following. The equation $D_{-}(k, 0)=0$ has only real roots; there are either

(a) three distinct roots $k_{01}(>0)$ and $k_{0 j}(<0) j=2,3$ or;

(b) one single root $k_{01}(>0)$ and a double root $K_{0}(<0)$, where $k_{02}<K_{0}<k_{03}$ or;

(c) a single root $k_{01}(>0)$.

These three cases arise accordingly as $u_{0} \leq$ or $\left.-(d / d k)(\sqrt{g k \tanh k h})\right]_{k=K_{0}}=u_{0}^{*}$ (say) (see Figure 4.1).

(I) Correspondingly, under the same condition on $u_{0}$, and for small $\nu$, we may conclude that the equation $D_{-}(k, v)=0$ possesses either (i) three roots $k_{j}, j=1,2,3$ or (ii) three roots $k_{1}, K_{2}, K_{3}$, the last 2 corresponding to the double root $K_{0}$, or (iii) only one root $k_{1}$; these roots $\left(k_{j} ; K_{\ell}\right)_{j=1,2,3, \ell=2,3}$ again reduce to the roots $k_{0 j}$ or $K_{0} j=1,2,3$ of $D_{-}(k, 0)=0$ in the respective cases, as $\nu \rightarrow 0+$.

(II) The equation $D_{+}(k, 0)=0$ has only one real root $k_{04}(>0)$ (Figure 4.2), and also complex roots outside the circle $|k|=\omega^{\prime} / u_{0}^{\prime}$ in the second and third quadrants of the $k$-plane for $-\pi / 4 \leq \operatorname{Im}(k) \leq \pi / 4$ in directions less than $2 \sin ^{-1}\left(1 / 2 \sqrt{u_{0}^{\prime} \omega^{\prime}}\right)$. For small $\nu$, and corresponding to these roots, the equation $D_{+}(k, v)=0$ then possesses, respectively, the root $k_{4}$ and a set of distinct complex roots which, however, leads to waves with enhanced damping due to presence of viscosity. Consequently, we ignore these latter roots and confine attention only to the root $k_{4}$ which again, as mentioned in (I), tends to $k_{04}$ as $v \rightarrow 0+$.

4.2. Exact form of propagating waves. Considering $C_{1}$ of (4.7) first for the case $u_{0}<u_{0}^{*}$, we use the result of Section 4.1(I) to rewrite it as

$$
C_{1}=C_{10}+C_{11}+C_{12}
$$

where

$$
\begin{gathered}
{\left[C_{10}, C_{11}, C_{12}\right]=\int_{-\infty}^{\infty} e^{-i k x} d k\left[N_{1}(k) \frac{\partial}{\partial k}\left\{\ln \frac{D_{-}(k)}{\Pi_{1}^{3}\left(k-k_{j}\right)}\right\}, \sum_{1}^{3} \frac{N_{1}(k)-N_{1}\left(k_{j}\right)}{k-k_{j}}, \sum_{1}^{3} \frac{N_{1}\left(k_{j}\right)}{k-k_{j}}\right],} \\
N_{1}(k)=\frac{P_{0}(k)}{2 D_{-}^{\prime}(k)} \cdot\left(\sigma-\frac{\sigma_{1}}{2}+\frac{i \sigma_{2}}{2}\right) .
\end{gathered}
$$


10 A study of the waves and boundary layers

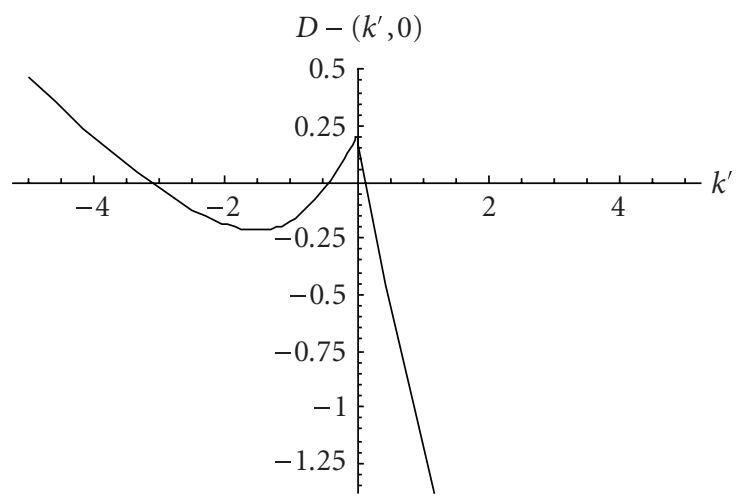

(a) Three distinct real zeros when $\omega^{\prime}=0.2$ and $u_{0}^{\prime}=0.5$.

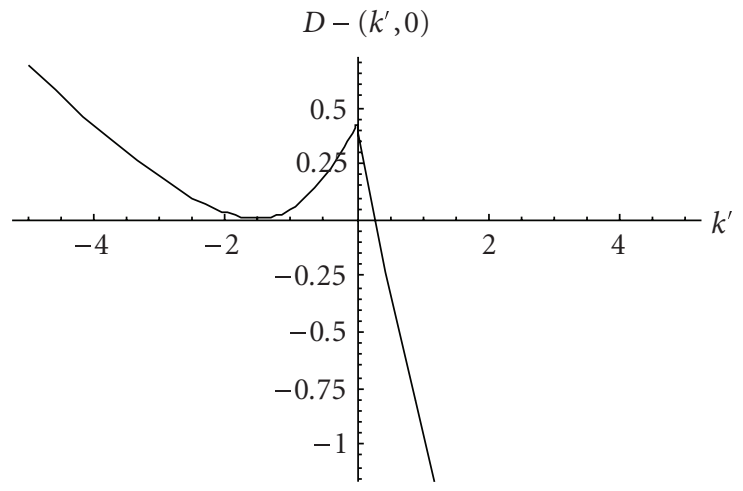

(b) Two real zeros, one single and the other repeated when $\omega^{\prime}=0.42$ and $u_{0}^{\prime}=0.5$.

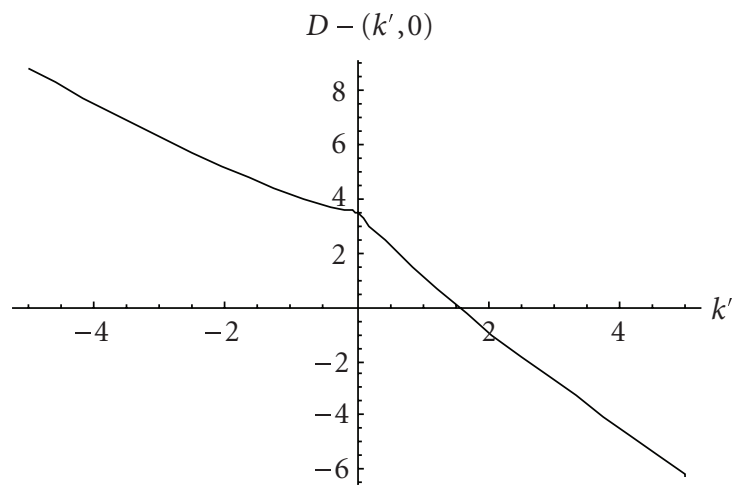

(c) Only a single real zero when $\omega^{\prime}=3.5$ and $u_{0}^{\prime}=1.5$.

Figure 4.1. Illustrative graphs of $D_{-}(k, 0)$ for the three cases of possible real zeros. 


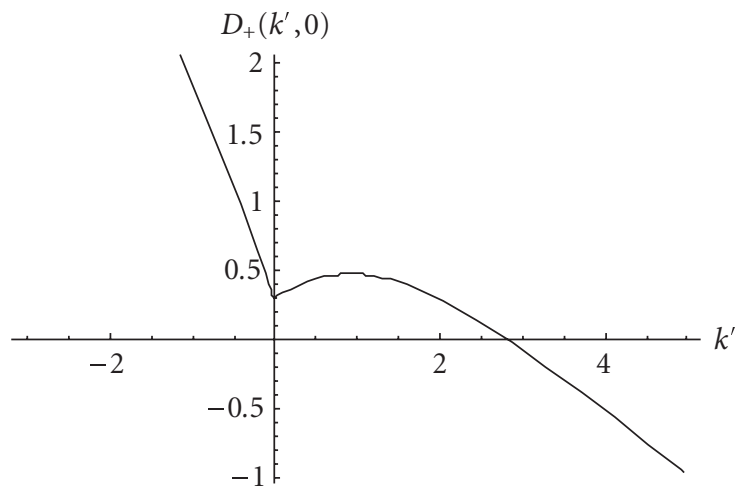

Figure 4.2. Illustrative graph of $D_{+}(k, 0)$ showing the occurrence of its real zero. $\omega^{\prime}=0.3$ and $u_{0}^{\prime}=0.7$.

Observing that $C_{10}$ is independent of $k_{j}$, and that the integrands of $C_{10}$ and $C_{11}$ are regular functions in the immediate vicinity (indented at $k=0$ ) of the real axis in the lower or upper half (for $x<$ or $>0$ ) of the complex $k$-plane, we conclude that $C_{10}$ and $C_{11}$ contribute only standing waves to $\zeta^{*}$ of the same frequency as that of the applied pressure.

For $C_{12}$, we use the result of Gradshteyn and Ryzhik [5, page 445, (2), (4), (6), (8)],

$$
\begin{aligned}
\int_{-\infty}^{\infty} \frac{e^{-i k x}}{k-k_{j}} d k=\pi( & \sqrt{-\frac{1}{k_{j}^{2}}} k_{j} \cosh \left[x \sqrt{-k_{j}^{2}}\right]-i \cos \left[x \sqrt{k_{j}^{2}}\right] \operatorname{Sign}[x] \\
& \left.+\left\{\sqrt{-k_{j}^{4}} \operatorname{sign}[x] \sin \left[x \sqrt{k_{j}^{2}}\right]\right\} \div \sqrt{-\frac{1}{k_{j}^{2}}} \cdot k_{j}^{3}+i \sinh \left[x \sqrt{-k_{j}^{2}}\right]\right) .
\end{aligned}
$$

It is then easily verified that the three components of $\zeta^{*}$ arising from three terms of $C_{12}$ each satisfies the wave equation

$$
\frac{\partial^{2} y}{\partial t^{2}}=c_{j}^{2} \frac{\partial^{2} y}{\partial x^{2}}, \quad \forall x, t, \text { where } c_{j}=\frac{\omega}{k_{j}}
$$

and hence each of these components represents a propagating wave with complex velocity $\omega / k_{j}$. Thus the progressive wave part of $\zeta^{*}$ arising from $C_{1}$ is

$$
\begin{aligned}
\zeta_{P 1}^{*}=\frac{e^{i \omega t}}{\sqrt{2 \pi} \rho g} \sum_{1}^{3} N_{1}\left(k_{j}\right) \pi( & \sqrt{-\frac{1}{k_{j}^{2}}} k_{j} \cosh \left[x \sqrt{-k_{j}^{2}}\right]-i \cos \left[x \sqrt{k_{j}^{2}}\right] \operatorname{Sign}[x] \\
& \left.+\left\{\sqrt{-k_{j}^{4}} \operatorname{sign}[x] \sin \left[x \sqrt{k_{j}^{2}}\right]\right\} \div \sqrt{-\frac{1}{k_{j}^{2}}} \cdot k_{j}^{3}+i \sinh \left[x \sqrt{-k_{j}^{2}}\right]\right) .
\end{aligned}
$$


For $u_{0}=u_{0}^{*}$, we have, in view of Section 4.1(I), three progressive waves again together with standing disturbances for all $x$ and $t$, the former being given by an expression of the same form as above with $K_{2}$ and $K_{3}$ replacing $k_{2}$ and $k_{3}$, respectively.

For $u_{0}>u_{0}^{*}$, Section $4.1(\mathrm{I})$ shows that $\zeta_{P 1}^{*}$ consists of only one progressive wave for all $x$ and $t$ given by the first term of $\zeta_{P 1}^{*}$ above.

$C_{2}$ of (4.8) may be treated similarly, and yields only one progressive wave $\zeta_{P 2}^{*}$ with complex velocity $\omega / k_{4}$ together with standing disturbances for all $x$ and $t, \zeta_{P 2}^{*}$ being of the same form as the first term of $\zeta_{P 1}^{*}$ with $N_{1}\left(k_{1}\right)$ replaced by $N_{2}\left(k_{4}\right)$, where $N_{2}(k)=$ $\left(P_{0}(k) / 2 D_{+}^{\prime}(k)\right) \cdot\left(\sigma_{1} / 2-\sigma+i \sigma_{2} / 2\right)$.

The final expression for the propagating part of $\zeta^{*}$ arising from $C_{1}$ and $C_{2}$ is then

$$
\begin{aligned}
& \zeta_{P}^{*}=\zeta_{P 1}^{*}+\zeta_{P 2}^{*}, \\
& =\frac{e^{i \omega t}}{\sqrt{2 \pi} \rho g} \sum_{1}^{3} N_{1}\left(k_{j}\right) \pi\left(\sqrt{-\frac{1}{k_{j}^{2}}} k_{j} \cosh \left[x \sqrt{-k_{j}^{2}}\right]-i \cos \left[x \sqrt{k_{j}^{2}}\right] \operatorname{Sign}[x]\right. \\
& \left.+\left\{\sqrt{-k_{j}^{4}} \operatorname{Sign}[x] \sin \left[x \sqrt{k_{j}^{2}}\right]\right\} \div \sqrt{-\frac{1}{k_{j}^{2}}} \cdot k_{j}^{3}+i \sinh \left[x \sqrt{-k_{j}^{2}}\right]\right) \\
& +N_{2}\left(k_{4}\right) \pi\left(\sqrt{-\frac{1}{k_{4}^{2}}} k_{4} \cosh \left[x \sqrt{-k_{4}^{2}}\right]-i \cos \left[x \sqrt{k_{4}^{2}}\right] \operatorname{Sign}[x]\right. \\
& \left.+\left\{\sqrt{-k_{4}^{4}} \operatorname{Sign}[x] \sin \left[x \sqrt{k_{4}^{2}}\right]\right\} \div \sqrt{-\frac{1}{k_{4}^{2}}} \cdot k_{4}^{3}+i \sinh \left[x \sqrt{-k_{4}^{2}}\right]\right) .
\end{aligned}
$$

It may be noted that the progressive wave part of $\zeta^{*}$ forms a wave-quartet when $u_{0} \leq u_{0}^{*}$, and a wave-duo when $u_{0}>u_{0}^{*}$, for all $x$ and $t$.

\section{Asymptotic expression of $\zeta^{*}$ for large $|x|$}

We first observe that the contributions of the standing waves associated with $\zeta_{P 1}^{*}$ and $\zeta_{P 2}^{*}$, as referred to in Section 4.2, to $\zeta^{*}$ are insignificant compared to the dominant terms of $\zeta_{P 1}^{*}$ and $\zeta_{P 2}^{*}$ for large $|x|$. The dominant term of the asymptotic expression of $\zeta^{*}$, for large $|x|$, denoted by $\zeta^{* *}$, can then be obtained from the asymptotic expansion of $\zeta_{P}^{*}$, given by (4.16). We have then

$$
\begin{gathered}
\zeta^{* *} \cong \frac{e^{i \omega t}}{\rho g}(2 \pi)^{1 / 2} i\left[\left\{N_{1}\left(k_{1}\right) e^{-i k_{1} x}+H\left(u_{0}^{*}-u_{0}\right) N_{1}\left(k_{3}\right) e^{-i k_{3} x}+N_{2}\left(k_{4}\right) e^{-i k_{4} x}\right\} H(x)\right. \\
\left.+H\left(u_{0}^{*}-u_{0}\right) N_{1}\left(k_{2}\right) e^{-i k_{2} x} H(-x)\right], \\
\zeta^{* *} \cong \frac{e^{i \omega t}}{\rho g}(2 \pi)^{1 / 2} i\left[\left\{N_{1}\left(k_{1}\right) e^{-i k_{1} x}+N_{1}\left(K_{3}\right) e^{-i K_{3} x}+N_{2}\left(k_{4}\right) e^{-i k_{4} x}\right\} H(x)\right. \\
\left.+N_{1}\left(K_{2}\right) e^{-i K_{2} x} H(-x)\right], \quad u_{0}=u_{0}^{*} .
\end{gathered}
$$


Although these two asymptotic expressions $\zeta^{* *}$ of $\zeta^{*}$ are in agreement in form with those of Pramanik [7], who performed an $O(\sqrt{\nu})$ analysis, there are sharp distinctions with regard to the content which arises mainly due to our $O(\nu)$ analysis of the problem. It will be seen later (Section 6) that a restriction to an $O(\sqrt{\nu})$ analysis makes the long-distance wave form inaccurate for large $h$.

\section{Cayley expansions of $k_{j}, N_{\ell}\left(k_{j}\right)$, and of $\zeta^{* *}$}

6.1. Expansions of $k_{j}$ and $N_{\ell}\left(k_{j}\right)$ in terms of their nonviscous values in a power series of $\nu^{1 / 2}$ or $v^{1 / 4}$ for small $\nu$. For actual as well as numerical calculations of the roots $\left(k_{j} ; K_{\ell}\right)$ of $D_{\mp}(k, v)=0$ and also of the wave amplitudes $N_{\ell}\left(k_{j}\right)$ for small $\nu$, we may use a result essentially due to Cayley (see Whittaker and Watson [12, page 147]). This enables us to express the above quantities in terms of the corresponding roots in the nonviscous problem in a power series of $v^{1 / 2}$ or $v^{1 / 4}$, respectively, for the two types of roots. To this purpose, we rewrite (4.9) and (4.10) in their equivalent forms ((a) and (b)) for the (first, second types) of roots, respectively,

(a) for

$$
\begin{gathered}
u_{0} \neq u_{0}^{*}, \\
{[(4.9),(4.10)] \longrightarrow \varphi_{\mp}(k)-\sqrt{v} f(k)=0, \quad f(k) \neq 0,}
\end{gathered}
$$

where

$$
\begin{gathered}
\varphi_{-}(k)=e^{-3 i \pi / 4} \sinh 2|k| h\left\{\sigma^{1 / 2}-\sqrt{-4\left(2 \sinh ^{2} 2 k h+\cosh 2 k h\right)\left(\omega-k u_{0}-\sigma\right)+\sigma}\right\}, \\
f(k)=2|k|\left(2 \sinh ^{2} 2 k h+\cosh 2 k h\right),
\end{gathered}
$$

and $\varphi_{+}(k)$ is obtained from $\varphi_{-}(k)$ on replacing $e^{-(3 i \pi) / 4}$ and $\left(\omega-k u_{0}-\sigma\right)$ by $e^{(3 i \pi) / 4}$ and $\left(\omega-k u_{0}+\sigma\right)$, respectively. We have (Cayley), for the three roots of (4.9) corresponding to the nonrepeated roots of $D_{-}(k, 0)=0$, and for the single root of $(4.10)$ corresponding to the root of $D_{+}(k, 0)=0$, the result

$$
k_{j}=k_{0 j}+\sqrt{v} k_{1 j}+v k_{2 j}+\cdots,
$$

where

$$
k_{1 j}=\frac{f\left(k_{0 j}\right)}{\varphi_{\mp}^{\prime}\left(k_{0 j}\right)}, \quad k_{2 j}=\frac{1}{2\left\{\varphi_{\mp}^{\prime}\left(k_{0 j}\right)\right\}^{3}}\left|\begin{array}{cc}
\varphi_{\mp}^{\prime} & f^{2} \\
\varphi_{\mp}^{\prime \prime} & \left(f^{2}\right)^{\prime}
\end{array}\right|_{k=k_{0 j}},
$$

upper sign for $u_{0}<u_{0}^{*}, \quad j=1,2,3$ the lower sign when $j=4$;

(b) for $u_{0}=u_{0}^{*}$, we replace (4.9) by the two equations

$$
\varphi_{1}(k)-(-1)^{\ell} \nu^{1 / 4} f_{1}(k)=0, \quad \ell=2,3,
$$

where

$$
\varphi_{1}(k)=\sqrt{\varphi_{-}(k)}, \quad f_{1}(k)=\sqrt{f(k)}
$$


so that by the same result cited above, the roots $K_{j}$ corresponding to a double root $K_{0} \equiv$ $K_{02} \equiv K_{03}$ may be expressed in a positive integral powers of $\nu^{1 / 4}$ as

$$
K_{j}=K_{0 j}+(-1)^{j} \nu^{1 / 4} K_{1 j}+\nu^{1 / 2} K_{2 j}+(-1)^{j} \nu^{3 / 4} K_{3 j}+\nu K_{4 j} \pm \cdots, \quad j=2,3,
$$

here $K_{02}=K_{03}=K_{0}$ and the coefficients $K_{i j}, i=1,2,3,4$ are given by the same formula as in the case (6.2) with $\varphi_{1}$ and $f_{1}$ replacing $\varphi_{-}$and $f$.

For $N_{\ell}\left(k_{j}\right)$, series expansions similar to the above follow from Cayley's general expansion formula [12, page 147]. The corresponding coefficients are the same as those given later (Section 6.2) in consideration with the asymptotic expression of $\zeta^{* *}$.

An interesting conclusion from the above expansions is that $\zeta_{P}^{*}$ in (4.16) is obtained correctly for large depth only when an $O(\nu)$ analysis of the problem is carried out instead of restricting it to an $O(\sqrt{v})$ (or, $\left.O\left(v^{3 / 4}\right)\right)$ one. For instance, in the case $u_{0}<u_{0}^{*}$, if $k h \gg$ $1 \varphi_{\mp} \sim(1 / 2) e^{\mp 3 i \pi / 4} e^{4 k h} \sqrt{\omega-k u_{0} \mp \sigma}$ and $f \sim|k| e^{4 k h}, k_{1 j} \rightarrow 0$ as $h \rightarrow \infty$ but $k_{2 j}$ does not tend to zero in the same limit. Hence if we keep $\zeta_{P}^{*}$ in (4.16) correct up to $O(\sqrt{v})$ terms, we get the paradoxical result that $\zeta_{P}^{*}$ is independent of $\nu$ as $h \rightarrow \infty$. The presence of $O(\nu)$ term in $\zeta_{P}^{*}$ shows that there is no such paradox, and leads to a correct value of $\zeta_{P}^{*}$ for large depths. When $u_{0}=u_{0}^{*}$, since $\varphi_{1}^{\prime}=\sqrt{\varphi_{-}^{\prime \prime} / 2}$, we may show similarly that $K_{\ell j} \rightarrow 0, \ell=1,2,3$, as $h \rightarrow \infty$ but $K_{4 j}$ does not tend to zero as $h \rightarrow \infty$; it follows as before that $\zeta_{P}^{*}$ for large depths is given correctly only when terms at least of the order $O(v)$ are retained. This justifies our assertion in Section 1 regarding the uniform validity of $\zeta_{P}^{*}$ for large $h$.

6.2. Cayley expansion of $\zeta^{* *}$. Use of Cayley expansion of $k_{j}$ and $N_{\ell}\left(k_{j}\right)$ as mentioned above helps us to visualize $\zeta^{* *}$ in a novel way. For then $\zeta^{* *}$ assumes the following form (a) or (b):

(a) when $u_{0} \neq u_{0}^{*}$,

$$
\zeta^{* *}=\frac{\sqrt{2 \pi} i}{\rho g}\left[\zeta_{0}+\sqrt{\nu} \zeta_{2}+\nu \zeta_{4}\right]
$$

where

$$
\begin{aligned}
& \zeta_{\ell}=H(x)\left\{M_{\ell} \times E_{1}\right\}+H(-x) H\left(u_{0}^{*}-u_{0}\right) N_{\ell 1}\left(k_{02}\right) e^{-i\left(k_{2} x-\omega t\right)}, \quad \ell=0,2,4, \\
& M_{\ell}=\left[\begin{array}{llll}
N_{\ell 1}\left(k_{01}\right) & H\left(u_{0}^{*}-u_{0}\right) & N_{\ell 1}\left(k_{03}\right) & N_{\ell 2}\left(k_{04}\right)
\end{array}\right]_{1 \times 3}, \quad \ell=0,2,4, \\
& E_{1}=\left[\begin{array}{lll}
e^{-i\left(k_{1} x-\omega t\right)} & e^{-i\left(k_{3} x-\omega t\right)} & e^{-i\left(k_{4} x-\omega t\right)}
\end{array}\right]^{T}, \\
& N_{0 j}(\alpha)=N_{j}(\alpha), \quad N_{2 j}=\frac{f(\alpha)}{\varphi_{\mp}^{\prime}(\alpha)} N_{j}^{\prime}(\alpha), \\
& N_{4 j}=\frac{1}{2\left\{\varphi_{\mp}^{\prime}(\alpha)\right\}^{3}}\left|\begin{array}{cc}
\varphi_{\mp}^{\prime} & \left(f^{2} N_{j}^{\prime}\right) \\
\varphi_{\mp}^{\prime \prime} & \left(f^{2} N_{j}^{\prime}\right)^{\prime}
\end{array}\right|_{k=\alpha}, \quad j=1,2,
\end{aligned}
$$

where $f$ and $\varphi_{\mp}$ are given by (6.4), $\varphi_{-}$is to be taken when $\alpha=k_{01}, k_{02}, k_{03}$, while $\varphi_{-}$of (6.4) is to be modified to $\varphi_{+}$as mentioned in Section 4.2 when $\alpha=k_{04}$; 
(b) when $u_{0}=u_{0}^{*}$,

$$
\zeta^{* *}=\frac{\sqrt{2 \pi} i}{\rho g}\left[\zeta_{0}^{\prime}+\nu^{1 / 4} \zeta_{1}^{\prime}+\nu^{1 / 2} \zeta_{2}^{\prime}+\nu^{3 / 4} \zeta_{3}^{\prime}+\nu \zeta_{4}^{\prime}\right]
$$

where

$$
\begin{aligned}
& \zeta_{\ell}^{\prime}=H(x)\left\{J_{\ell} \times E_{2}\right\}+H(-x) J_{\ell 1}\left(K_{02}\right) e^{-i\left(k_{2} x-\omega t\right)}, \quad \ell=0,1,2,3,4 \text {, }
\end{aligned}
$$

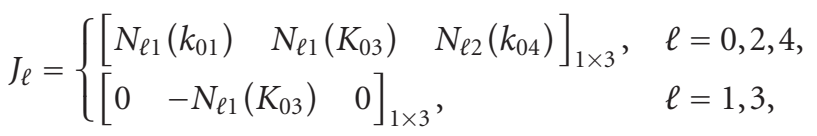

$$
\begin{aligned}
& E_{2}=\left[\begin{array}{lll}
e^{-i\left(k_{1} x-\omega t\right)} & e^{-i\left(K_{3} x-\omega t\right)} & e^{-i\left(k_{4} x-\omega t\right)}
\end{array}\right]^{T}, \\
& N_{0 j}(\alpha)=N_{j}(\alpha) \text {, } \\
& N_{2 j}=\frac{1}{2\left\{\varphi_{\mp}^{\prime}(\alpha)\right\}^{3}}\left|\begin{array}{ll}
\varphi_{\mp}^{\prime} & \left(f_{1}^{2} N_{j}^{\prime}\right) \\
\varphi_{\mp}^{\prime \prime} & \left(f_{1}^{2} N_{j}^{\prime}\right)^{\prime}
\end{array}\right|_{k=\alpha} \quad \text { when } \alpha=k_{01}, k_{04}, j=1,2 \text {, } \\
& N_{11}=\frac{f_{1}\left(K_{03}\right)}{\varphi_{1}^{\prime}\left(K_{03}\right)} N_{j}^{\prime}\left(K_{03}\right) \text {, and so forth. }
\end{aligned}
$$

We note that each $\zeta_{j}$ is a wave-quartet or a wave-duo and the terms of (6.9) and (6.11) are scaled down in powers of $\nu^{1 / 2}$ or $v^{1 / 4}$, respectively. Further the exponential parts of the four or two components of $\zeta_{j}$ remain invariant for all $j$ while the nonexponential parts are independent of $v$, these being functions of $k_{o j}$ only. The strength of these expansions becomes more evident as we proceed to make a numerical study of the wave motion and also compare these solutions in exact form with those of their asymptotic counterpart.

\section{Cross-vertical vorticity and boundary layers and their asymptotic expansion}

In what follows, we show how to obtain approximate expressions of the thickness of the boundary layers which are formed at the surface and at the bottom because of the motion having a high Reynolds number $\left(\sqrt{g h^{3}}\right) / \nu$. We note that viscosity generates only the crossvertical vorticity $\omega_{z}$ which is obtained from (2.5) with the help of (2.6) and (2.9) as

$$
\begin{aligned}
& \omega_{z}=\frac{1}{(2 \pi) 2^{3 / 2} i \rho} \int_{-\infty}^{\infty} \operatorname{sgn} k e^{-i k x} d k \int_{c-i \infty}^{c+i \infty} \widetilde{P}_{0} e^{s t} d s \\
& \times\left(\left[2 k^{2}\left(m-i k^{2}\right)\{|k| \sinh m(y+h) \operatorname{sech} m h-m \cosh m(y+h) \operatorname{sech} m h \tanh |k| h\}\right.\right. \\
& +k^{2}\left(k^{2}+m^{2}\right)(1-i)\{m \sinh |k|(y+h) \sec h|k| h \\
& -|k| \cosh |k|(y+h) \sec h|k| h \tanh m h\} \\
& -|k|\left(k^{2}+m^{2}\right)\left(m^{2}-i k^{2}\right) \sinh m y \sec h m h \sec h|k| h \\
& \left.\left.-2 k^{4}(1-i) m \sinh |k| y \operatorname{sech} m h \sec h|k| h\right]\right) \div\left(\nu^{-3 / 2} f\left(\nu^{1 / 2}\right)\right) \text {. }
\end{aligned}
$$


If $\delta_{s}, \delta_{b}$ are the thicknesses of the surface and bottom boundary layers, $\omega_{z}=0$ for $\delta_{s} \leq$ $|y| \leq h-\delta_{b}$, because the motion is irrotational just outside the boundary layers. An approximate value of $\delta_{s}$ may thus be obtained as

$$
\delta_{s}=-\frac{\left.\omega_{z}\right)_{y=0}}{\left.\partial \omega_{z} / \partial y\right]_{y=0}}
$$

with a similar expression for $\delta_{b}$. To obtain the expression for thickness $\delta_{s}^{*}$ of the layer below the leading steady-state wave, we analyze the expressions for $\left.\omega_{z}^{*}\right)_{y=0}$ and $\partial \omega_{z}^{*} /$ $\partial y]_{y=0}$ in the same way as done for the determination of $\zeta$ correct up to $O(\nu)$ terms. This gives, possibly for further restrictions on the order of $P_{0}(k)$ as $|k| \rightarrow \infty$, the following:

$$
\begin{gathered}
\left.\left.\omega_{z}^{*}\right)_{y=0} \cong \frac{e^{i \omega t}}{\sqrt{2 \pi} \rho g}\left(C_{4}+C_{5}\right), \quad \frac{\partial \omega_{z}^{*}}{\partial y}\right]_{y=0} \cong \frac{e^{i \omega t}}{\sqrt{2 \pi} \rho g}\left(C_{6}+C_{7}\right), \\
\delta_{s}^{*}=-\frac{\left.\omega_{z}^{*}\right)_{y=0}}{\left.\partial \omega_{z}^{*} / \partial y\right]_{y=0}},
\end{gathered}
$$

here

$$
\begin{aligned}
& C_{4}=\int_{-\infty}^{\infty} \frac{P_{0}(k) S_{1}(k)}{-\sigma+\sigma_{1}+\omega-k u_{0}-i \sigma_{2}} e^{-i k x} d k, \\
& C_{5}=\int_{-\infty}^{\infty} \frac{-P_{0}(k) \bar{S}_{1}(k)}{\sigma-\sigma_{1}+\omega-k u_{0}-i \sigma_{2}} e^{-i k x} d k, \\
& C_{6}=\int_{-\infty}^{\infty} \frac{P_{0}(k) S_{2}(k)}{-\sigma+\sigma_{1}+\omega-k u_{0}-i \sigma_{2}} e^{-i k x} d k, \\
& C_{7}=\int_{-\infty}^{\infty} \frac{-P_{0}(k) \bar{S}_{2}(k)}{\sigma-\sigma_{1}+\omega-k u_{0}-i \sigma_{2}} e^{-i k x} d k,
\end{aligned}
$$

where $S_{1}(k)$ and $S_{2}(k)$ are given by

$$
\begin{aligned}
& S_{1}(k)=k^{2} {\left[\left(\frac{3 \sigma \sigma_{2}}{2}+\sigma^{2}-\sigma_{1}^{2}-\frac{3 \sigma \sigma_{1}}{2}\right)+i\left(\frac{3 \sigma \sigma_{2}}{2}-\sigma^{2}-\sigma_{1}^{2}+\frac{3 \sigma \sigma_{1}}{2}\right)\right], } \\
& S_{2}(k)=\frac{k^{2} \sigma^{3 / 2}}{2^{1 / 2}}\left[\left\{4\left(\sigma_{2}-\sigma_{1}\right)+2 \sigma+\frac{3 \sigma_{1}^{2}}{4}+k^{2} \tanh |k| h+5|k| \sigma_{1} \nu^{1 / 2}\right\}\right. \\
&\left.+i\left\{4\left(\sigma_{2}+\sigma_{1}\right)-2 \sigma-\frac{3 \sigma_{1}^{2}}{4}-7 k^{2} \tanh |k| h+|k|\left(5 \sigma_{1}-2 \sigma\right)\right\}\right] .
\end{aligned}
$$


Denoting the asymptotic value of $\left.\omega_{z}^{*}\right)_{y=0}$ and $\left.\partial \omega_{z}^{*} / \partial y\right]_{y=0}$ at large distances by $\omega_{z}^{* *}$ and $\partial \omega_{z}^{* *} / \partial y$, we have

$$
\begin{gathered}
\omega_{z}^{* *} \cong \frac{e^{i \omega t}}{\rho g}(2 \pi)^{1 / 2} i\left[\left\{G_{1}\left(k_{1}\right) e^{-i k_{1} x}+H\left(u_{0}^{*}-u_{0}\right) G_{1}\left(k_{3}\right) e^{-i k_{3} x}+G_{2}\left(k_{4}\right) e^{-i k_{4} x}\right\} H(x)\right. \\
\left.+H\left(u_{0}^{*}-u_{0}\right) G_{1}\left(k_{2}\right) e^{-i k_{2} x} H(-x)\right],
\end{gathered}
$$

where

$$
\begin{gathered}
G_{1}(\alpha)=\left[\frac{P_{0}(k) S_{1}(k)}{\left\{-\sigma+\sigma_{1}+\omega-k u_{0}-i \sigma_{2}\right\}^{\prime}}\right]_{k=\alpha}, \\
G_{2}\left(k_{4}\right)=\left[\frac{-P_{0}(k) \bar{S}_{1}(k)}{\left\{\sigma-\sigma_{1}+\omega-k u_{0}-i \sigma_{2}\right\}^{\prime}}\right]_{k=k_{4}}, \\
\frac{\partial \omega_{z}^{* *}}{\partial y} \cong \frac{e^{i \omega t}}{\rho g}(2 \pi)^{1 / 2} i\left[\left\{H_{1}\left(k_{1}\right) e^{-i k_{1} x}+H\left(u_{0}^{*}-u_{0}\right) H_{1}\left(k_{3}\right) e^{-i k_{3} x}+H_{2}\left(k_{4}\right) e^{-i k_{4} x}\right\} H(x)\right. \\
\left.+H\left(u_{0}^{*}-u_{0}\right) H_{1}\left(k_{2}\right) e^{-i k_{2} x} H(-x)\right],
\end{gathered}
$$

where

$$
\begin{gathered}
H_{1}(\alpha)=\left[\frac{P_{0}(k) S_{2}(k)}{\left\{-\sigma+\sigma_{1}+\omega-k u_{0}-i \sigma_{2}\right\}^{\prime}}\right]_{k=\alpha}, \\
H_{2}\left(k_{4}\right)=\left[\frac{-P_{0}(k) \bar{S}_{2}(k)}{\left\{\sigma-\sigma_{1}+\omega-k u_{0}-i \sigma_{2}\right\}^{\prime}}\right]_{k=k_{4}},
\end{gathered}
$$

here accent $\left({ }^{\prime}\right)$ denotes $d / d k$.

By applying Cayley's expansions again as in the case of (6.9), we can find the asymptotic expansion of $\omega_{z}^{* *}$ and $\partial \omega_{z}^{* *} / \partial y$ at large distances in powers of $\nu^{1 / 2}$ as when $u_{0} \neq u_{0}^{*}$,

$$
\omega_{z}^{* *}=\frac{\sqrt{2 \pi} i}{\rho g}\left[\omega_{0}+\sqrt{\nu} \omega_{2}+\nu \omega_{4}\right]
$$

where

$$
\begin{gathered}
\omega_{\ell}=H(x)\left\{M_{\ell} \times E_{1}\right\}+H(-x) H\left(u_{0}^{*}-u_{0}\right) N_{\ell 1}\left(k_{02}\right) e^{-i\left(k_{2} x-\omega t\right)}, \quad \ell=0,2,4, \\
M_{\ell}=\left[\begin{array}{lll}
G_{\ell 1}\left(k_{01}\right) & H\left(u_{0}^{*}-u_{0}\right) G_{\ell 1}\left(k_{03}\right) & G_{\ell 2}\left(k_{04}\right)
\end{array}\right]_{1 \times 3}, \quad \ell=0,2,4, \\
E_{1}=\left[\begin{array}{lll}
e^{-i\left(k_{1} x-\omega t\right)} & e^{-i\left(k_{3} x-\omega t\right)} & e^{-i\left(k_{4} x-\omega t\right)}
\end{array}\right]^{T}, \\
G_{0}(\alpha)=G_{j}(\alpha), \quad G_{2 j}=\frac{f(\alpha)}{\varphi_{\mp}^{\prime}(\alpha)} G_{j}^{\prime}(\alpha), \\
G_{4 j}=\frac{1}{2\left\{\varphi_{\mp}^{\prime}(\alpha)\right\}^{3}}\left|\begin{array}{ll}
\varphi_{\mp}^{\prime} & \left(f^{2} G_{j}^{\prime}\right) \\
\varphi_{\mp}^{\prime \prime} & \left(f^{2} G_{j}^{\prime}\right)^{\prime}
\end{array}\right|_{k=\alpha}, \quad j=1,2,
\end{gathered}
$$


when $u_{0} \neq u_{0}^{*}$,

$$
\frac{\partial \omega_{z}^{* *}}{\partial z}=\frac{\sqrt{2 \pi} i}{\sqrt{v} \rho g}\left[\omega_{0}+\sqrt{v} \omega_{2}+v \omega_{4}\right]
$$

where

$$
\begin{gathered}
\tilde{\omega}_{\ell}=H(x)\left\{M_{\ell} \times E_{1}\right\}+H(-x) H\left(u_{0}^{*}-u_{0}\right) N_{\ell 1}\left(k_{02}\right) e^{-i\left(k_{2} x-\omega t\right)}, \quad \ell=0,2,4, \\
M_{\ell}=\left[\begin{array}{lll}
H_{\ell 1}\left(k_{01}\right) & H\left(u_{0}^{*}-u_{0}\right) H_{\ell 1}\left(k_{03}\right) & H_{\ell 2}\left(k_{04}\right)
\end{array}\right]_{1 \times 3}, \quad \ell=0,2,4, \\
E_{1}=\left[\begin{array}{lll}
e^{-i\left(k_{1} x-\omega t\right)} & e^{-i\left(k_{3} x-\omega t\right)} & e^{-i\left(k_{4} x-\omega t\right)}
\end{array}\right]^{T}, \\
H_{0}(\alpha)=H_{j}(\alpha), \quad H_{2 j}=\frac{f(\alpha)}{\varphi_{\mp}^{\prime}(\alpha)} H_{j}^{\prime}(\alpha), \\
H_{4 j}=\frac{1}{2\left\{\varphi_{\mp}^{\prime}(\alpha)\right\}^{3}}\left|\begin{array}{ll}
\varphi_{\mp}^{\prime} & \left(f^{2} H_{j}^{\prime}\right) \\
\varphi_{\mp}^{\prime \prime} & \left(f^{2} H_{j}^{\prime}\right)^{\prime}
\end{array}\right|_{k=\alpha}, j=1,2,
\end{gathered}
$$

where $f$ and $\varphi_{\mp}$ are given by (6.4), $\varphi_{-}$is to be taken when $\alpha=k_{01}, k_{02}, k_{03}$, while $\varphi_{-}$of (6.4) is to be modified to $\varphi_{+}$as mentioned in Section 4.2 when $\alpha=k_{04}$.

From (7.2), we have by the help of (7.9) the approximate thickness of the surface boundary layer in the asymptotic case as

$$
\delta_{s}^{* *}=\frac{\omega_{0}}{\widetilde{\omega}_{0}} \sqrt{\nu}+\frac{\omega_{2} \tilde{\omega}_{0}-\omega_{0} \tilde{\omega}_{2}}{\tilde{\omega}_{0}^{2}} \nu .
$$

We note that $\delta_{s}^{*}$ and $\delta_{s}^{* *}$ are both independent of $t$, in other words, the shape of the steady-state boundary layer is not affected by the periodicity of the surface forcing.

\section{Physical conclusions with numerical illustrations of the exact and asymptotic forms of steady-state progressive waves and boundary layer thickness}

Here we mention some physical features of the exact steady-state progressive waves exact and asymptotic as expressed by the result derived in Sections 4.2 and 6.2. We also undertake a numerical evaluation of (4.16), (6.9), and (6.11) to illustrate these features along with that of the surface boundary layer thicknesses in the exact and asymptotic forms as shown in (7.3) and (7.13). For this, we assume a general parabolic distribution of applied surface pressure of the form

$$
p_{0}(x)= \begin{cases}A\left(1-x^{2}\right)^{\lambda-1} H(t) & \text { when }|x| \leq 1, \lambda>\frac{15}{4}, \quad H(t) \text { is the Heaviside unit function, } \\ 0 & \text { when }|x|>1,\end{cases}
$$

which gradually falls from a maximum value at the origin to zero on a finite strip of the free surface. Clearly then, we have $P_{0}(k)=\left\{8 \sqrt{2} A \Gamma(1 / 2) J_{4}(k)\right\} / k^{4}$, taking $\lambda=9 / 2$. 


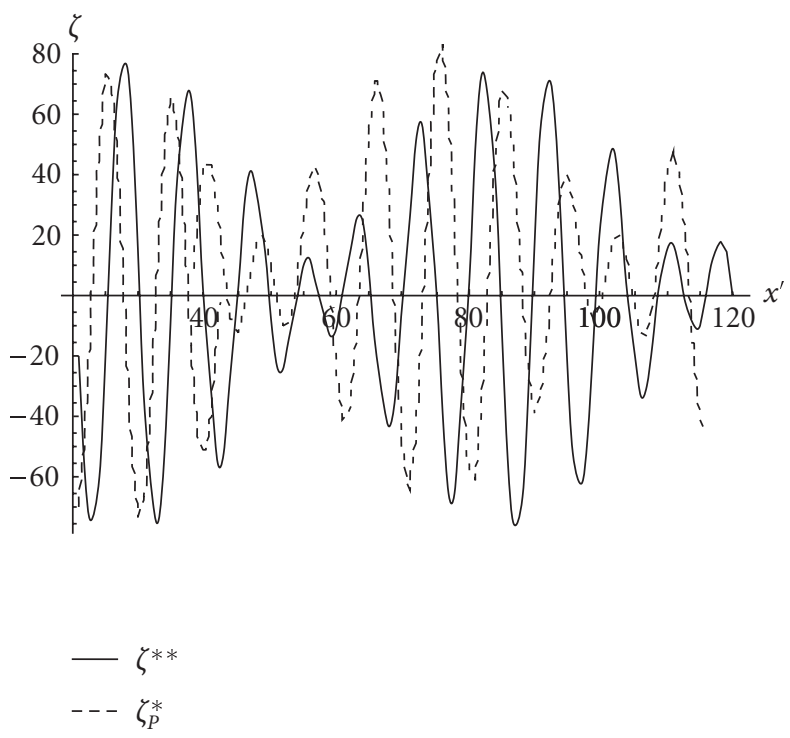

Figure 8.1

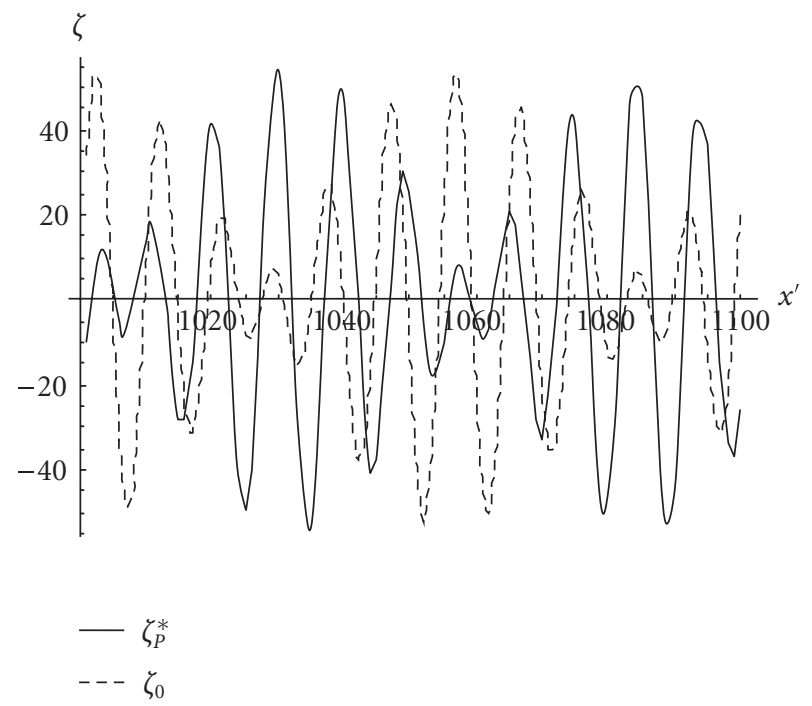

Figure 8.2

We first provide a numerical evaluation of the progressive part of the exact wave form represented by $\zeta_{P}^{*}(4.16)$ when $u_{0}>u_{0}^{*}$ for moderate distances. If the asymptotic wave form (6.9) is also computed under the same situation, we notice a close resemblance between the two wave forms albeit with a lateral shift (Figure 8.1). This indicates the 


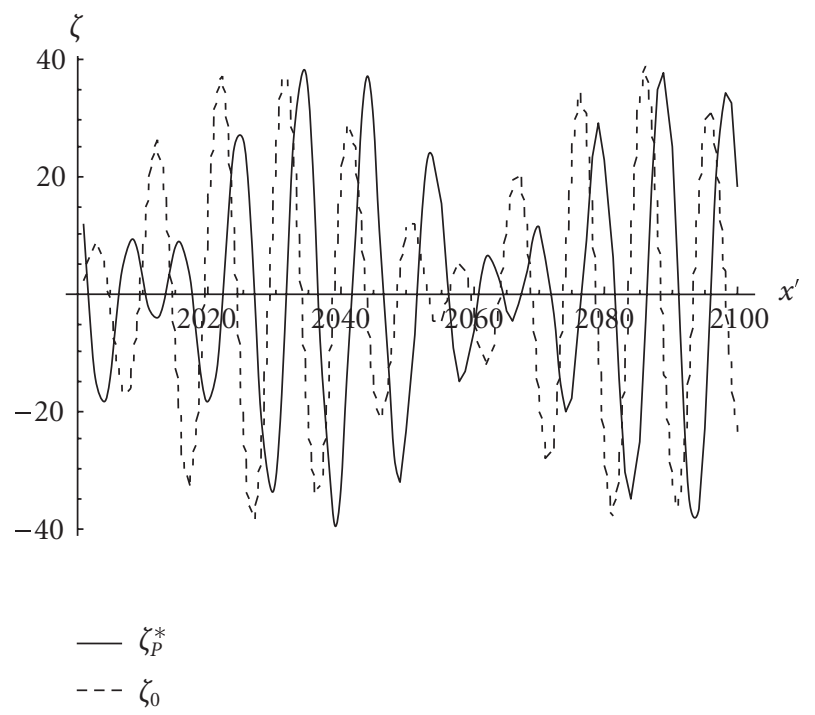

Figure 8.3

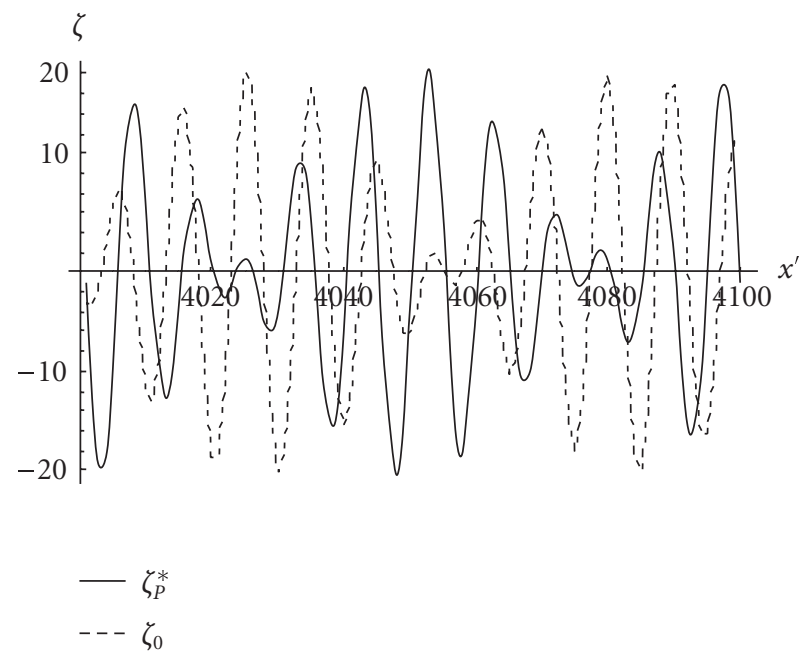

Figure 8.4

role of the damping factor in obliterating quickly the differences between the two with increasing distances.

Next we show that at large distances, the leading term $\zeta_{0}$ of the Cayley asymptotic expansion (6.9) is alone a sufficient approximation of $\zeta_{P}^{*}$, which is the exact progressive wave component of $\zeta^{*}$. Another observation of interest is that for any specified range, 


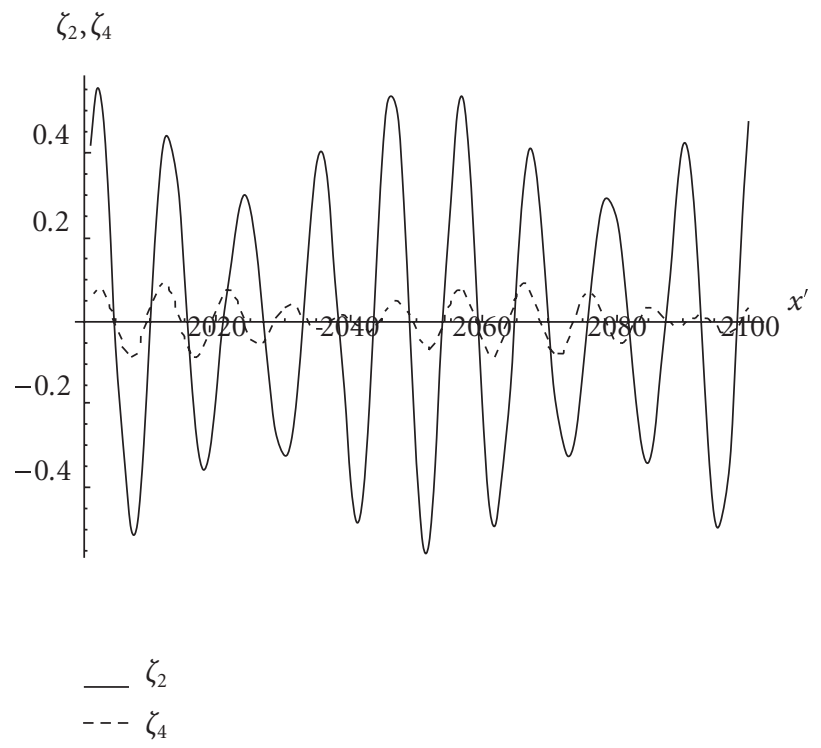

Figure 8.5

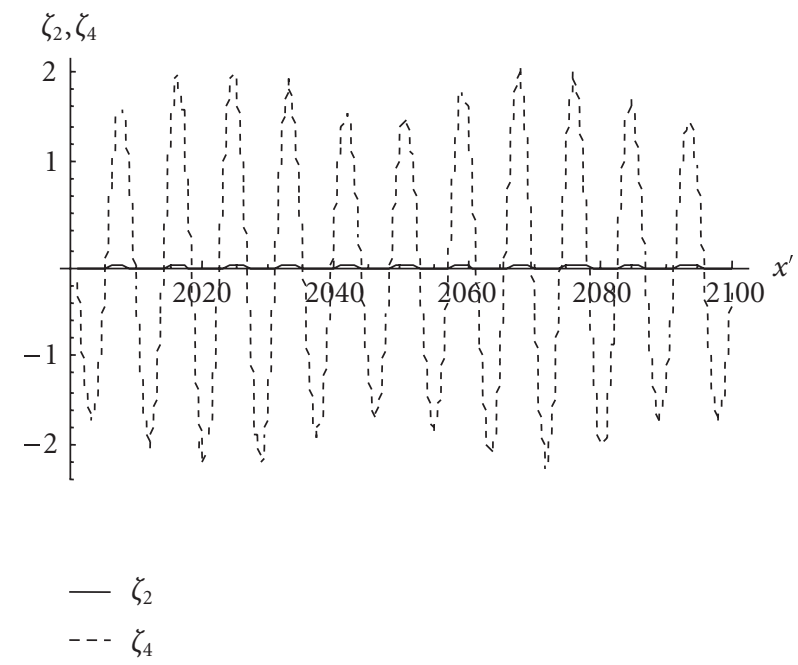

Figure 8.6. When the depth is ten times, and as a result we have taken $\omega^{\prime}=6.3 \times \sqrt{10}, u_{0}^{\prime}=(1 / \sqrt{10}) \times$ $10, v^{\prime}=\left(1 / 10^{3 / 2}\right) \times 0.0002$.

when the distances are doubled, the wave amplitudes are on the average approximately halved. These are shown in Figures 8.2 to 8.4.

For the long-distance asymptotics (6.9), we note that the other two components $\sqrt{\nu} \zeta_{2}$ and $\nu \zeta_{4}$ are insignificant compared to $\zeta_{0}$. However $\zeta_{2}$ and $\zeta_{4}$ are illustrated separately in Figure 8.5 to emphasize that ultimately $\zeta_{4}$ dominates $\zeta_{2}$ which diminishes to zero with 


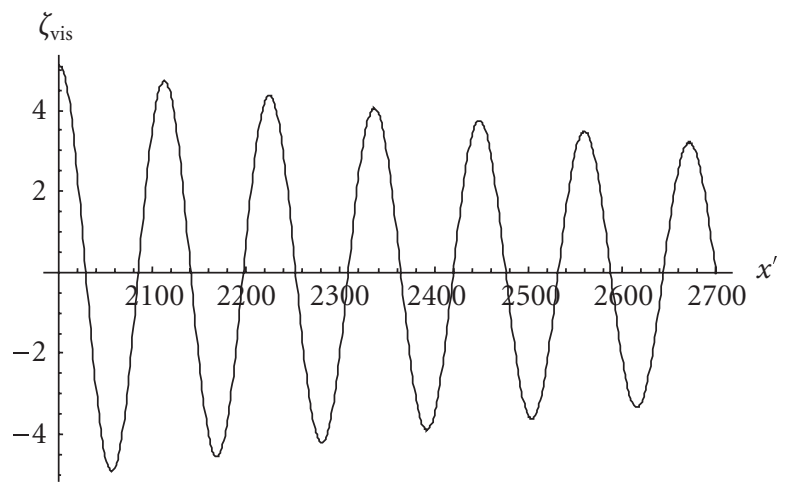

Figure 8.7. The form of dominant $\zeta_{\text {vis }}$, near the critical value of $u_{0}^{\prime} . \omega^{\prime}=0.1, u_{0}^{\prime}=0.8,\left(u_{0}^{\prime *}=0.787\right)$, $v^{\prime}=0.0002$.

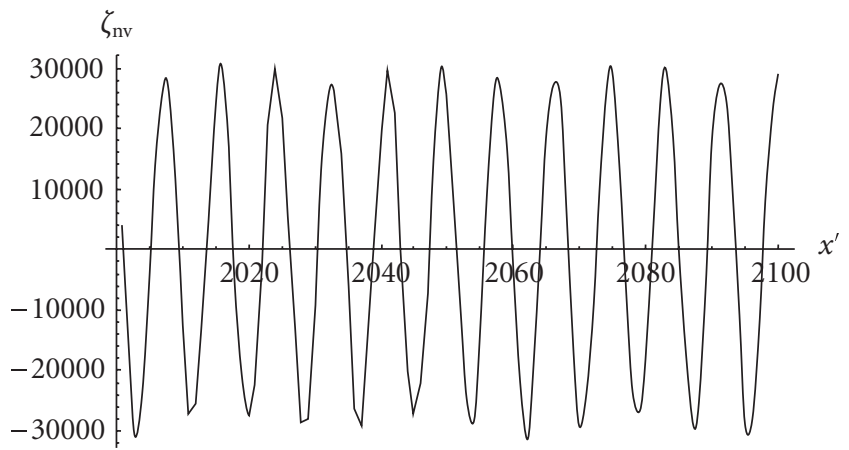

Figure 8.8. The situation of nonviscous $\zeta$, near the critical value of $u_{0}^{\prime} . \omega^{\prime}=0.1, u_{0}^{\prime}=0.8,\left(u_{0}^{\prime *}=\right.$ $0.787), v^{\prime}=0.0002$.

increasing ocean depth (Figure 8.6). Indeed the orders of magnitude of $\zeta_{2}$ and $\zeta_{4}$ are reversed as the ocean depth increases or viscosity diminishes, this also signifies the importance of an $O(\nu)$ analysis of the problem. In Figures 8.7 and 8.8, we compare the wave-heights in the vicinity of a certain critical speed $u_{o}^{*}$ of the running stream in the viscous and nonviscous fluids, respectively. The latter demonstrates the exceedingly large wave heights which are known to occur in this case; the former however illustrates the finiteness of the wave-amplitude in the same. In Figure 8.9, a comparison sketch of the waves is shown in the viscous and nonviscous case, and the viscous wave damping is well observed here.

We next determine the steady-state surface boundary layer thickness $\delta_{s}^{*}$ from (7.3) by the same numerical process as used for evaluating the exact progressive wave forms in Section 4.2. The corresponding variation of thickness of the surface boundary layer with distance, for fixed depth and time, is shown in Figure 8.10 along with that of the exact steady-state progressive wave form $\zeta_{P}^{*}$ evaluated earlier. In conclusion, we would like to 


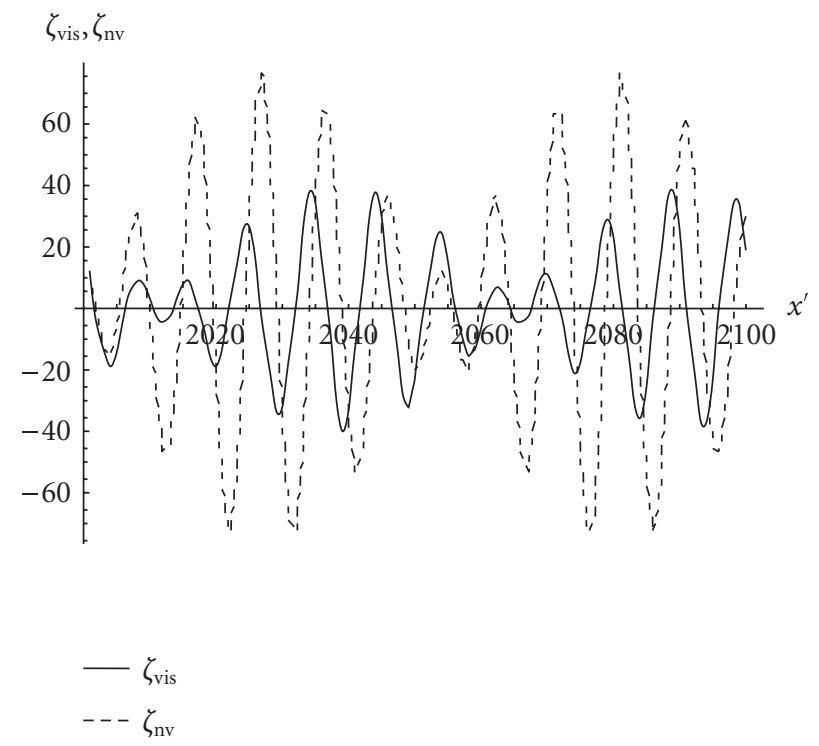

Figure 8.9

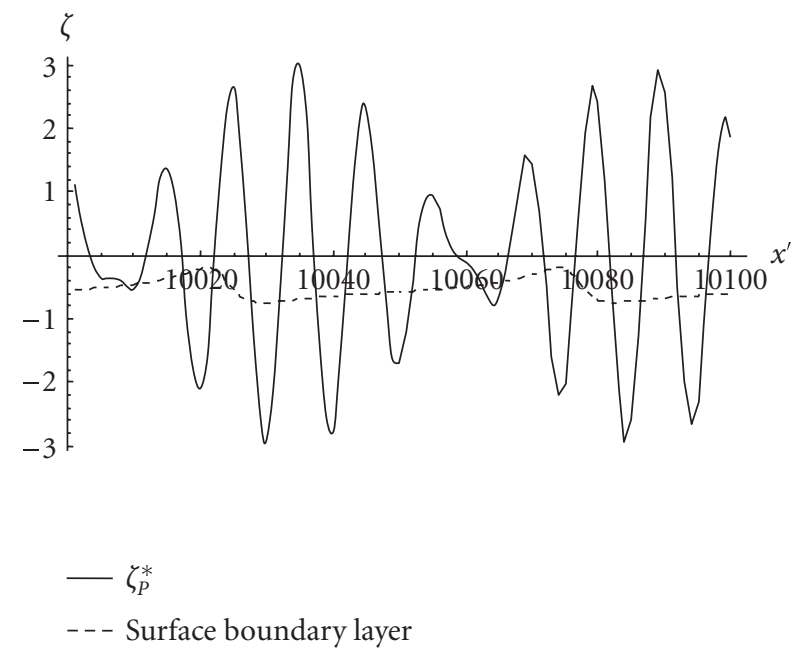

Figure 8.10

mention the following dimensionless parameters:

$$
\omega^{\prime}=\omega \sqrt{\frac{h}{g}}, \quad u_{0}^{\prime}=\frac{u_{0}}{\sqrt{g h}}, \quad v^{\prime}=\frac{v}{\sqrt{g h^{3}}}, \quad x^{\prime}=\frac{x}{h}, \quad k^{\prime}=k h,
$$


which are being used while performing the computational study of the problem for Figures $4.1,4.2$, and $8.1-8.10$.

In Figures 8.1-8.5 and 8.9-8.10, the values taken for the nondimensional parameters are $\omega^{\prime}=6.3, u_{0}^{\prime}=10, v^{\prime}=0.0002$.

\section{Acknowledgments}

The author is deeply indebted to Professor A. R. Sen of the Department of Mathematics, Jadavpur University, Calcutta, for his help and suggestions during the preparation of this paper. The author is also grateful to the referees for suggesting some improvements, which have since been incorporated in the paper.

\section{References}

[1] A. Bandyopadhyay, Effects of viscosity on linear gravity waves due to surface disturbances in water of finite depth, ZAMM. Zeitschrift für Angewandte Mathematik und Mechanik 83 (2003), no. 3, 205-213.

[2] J. T. Beale, Large-time regularity of viscous surface waves, Archive for Rational Mechanics and Analysis 84 (1984), no. 4, 307-352.

[3] N. Bleistein and R. A. Handelsman, Asymptotic Expansions of Integrals, Holt, Rinehart \& Winston, New York, 1975.

[4] U. T. Ehrenmark and G. L. Body, The initial-value problem on a plane beach, The Quarterly Journal of Mechanics and Applied Mathematics 54 (2001), no. 3, 389-411.

[5] I. S. Gradshteyn and I. M. Ryzhik, Table of Integrals, Series, and Products, 4th ed., Academic Press, New York, 1965.

[6] A. K. Nikitin and E. N. Potetyunko, The three-dimensional Cauchy-Poisson problem for waves on the surface of a viscous liquid of finite depth, Soviet Physics. Doklady 12 (1967), no. 5, 422-424.

[7] A. K. Pramanik, Waves due to an oscillatory pressure on a shallow viscous stream, The Quarterly Journal of Mechanics and Applied Mathematics 26 (1973), no. 2, 193-204.

[8] A. Prosperetti, Viscous effects on small-amplitude surface waves, Physics of Fluids 19 (1976), no. 2, 195-203.

[9] I. N. Sneddon, The Use of Integral Transforms, McGraw-Hill, New York, 1972.

[10] C. J. Tranter, Integral Transforms in Mathematical Physics, Methuen, London, 1951.

[11] J. V. Wehausen and E. V. Laitone, Surface waves, Handbuch der Physik, Vol. 9, Part 3, Springer, Berlin, 1960, pp. 446-778.

[12] E. T. Whittaker and G. N. Watson, A Course of Modern Analysis, Cambridge University Press, Cambridge, 1920.

Arghya Bandyopadhyay: Department of Mathematics, Khalisani College, Hooghly 712 138, India E-mail address: b.arghya@gmail.com 


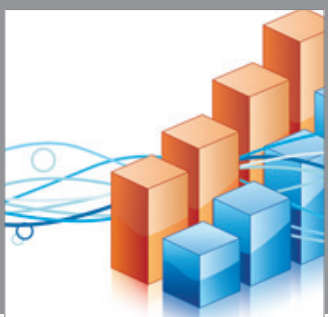

Advances in

Operations Research

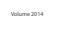

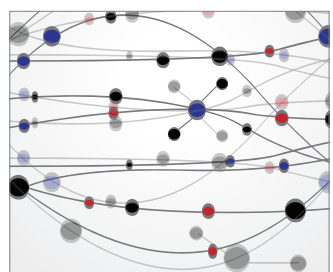

\section{The Scientific} World Journal
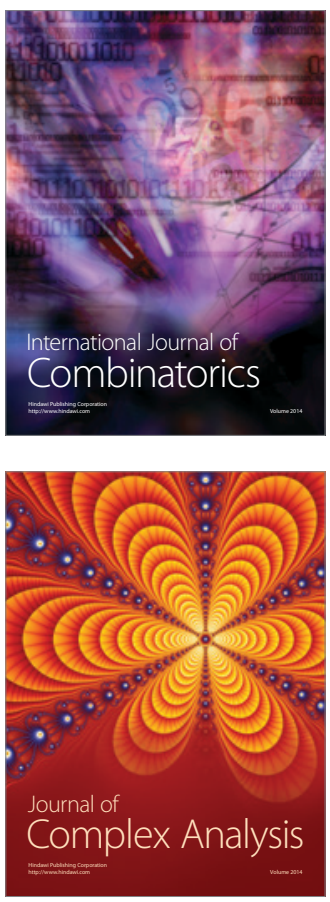

International Journal of

Mathematics and

Mathematical

Sciences
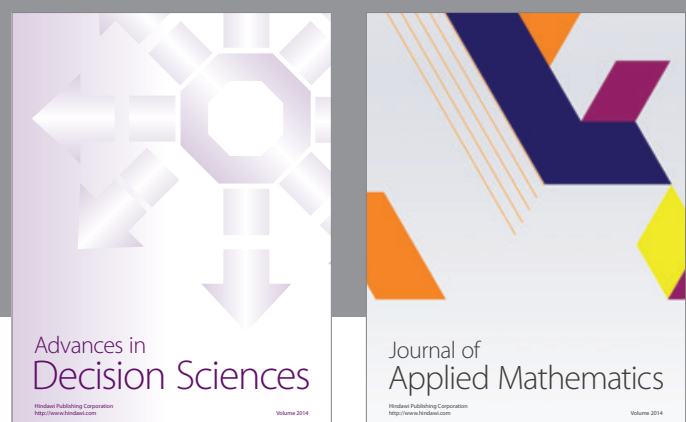

Journal of

Applied Mathematics
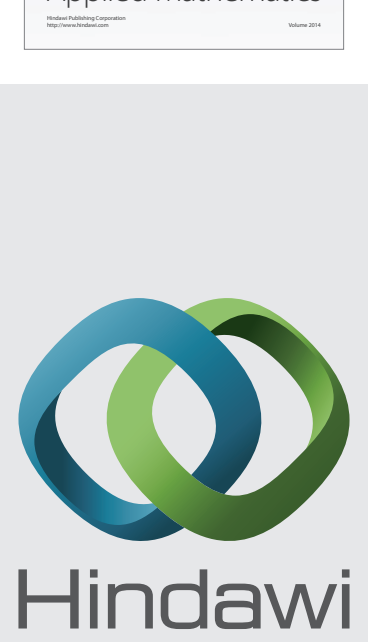

Submit your manuscripts at http://www.hindawi.com
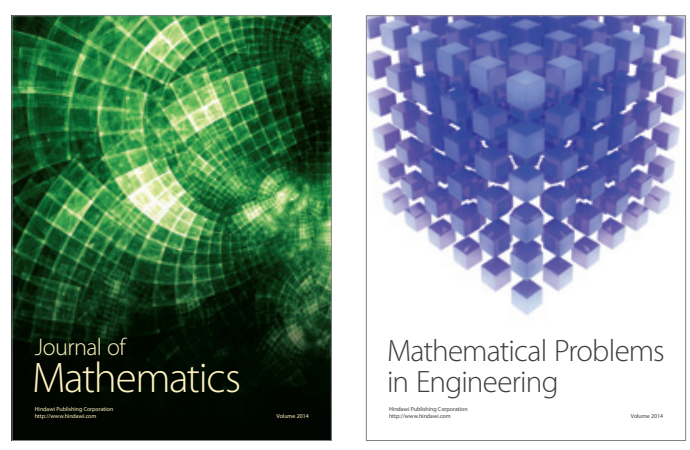

Mathematical Problems in Engineering
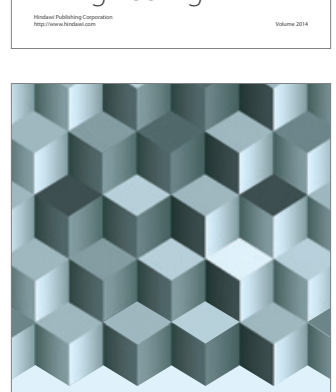

Journal of

Function Spaces
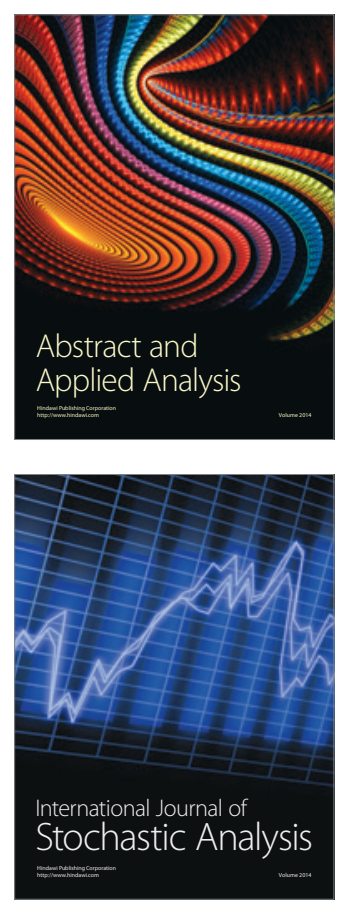

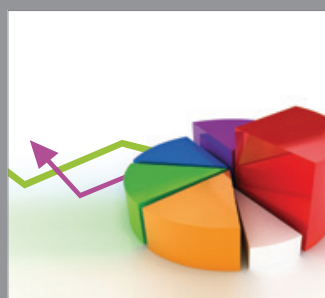

ournal of

Probability and Statistics

Promensencen
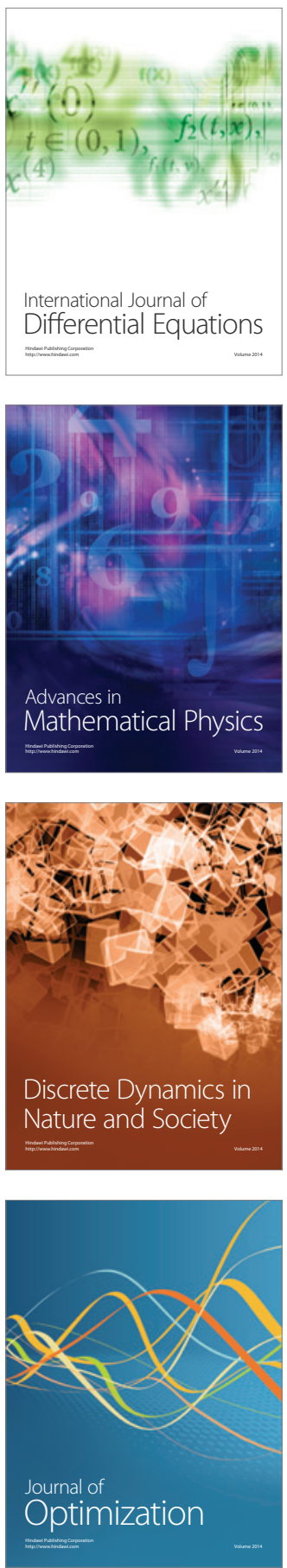